\title{
A Translation-Invariant Renormalizable Non-Commutative Scalar Model
}

\author{
R. Gurau ${ }^{1}$, J. Magnen ${ }^{2}$, V. Rivasseau ${ }^{1}$, A. Tanasa ${ }^{3,4}$ \\ 1 Laboratoire de Physique Théorique, CNRS UMR 8627, bât. 210, Université Paris XI, \\ 91405 Orsay Cedex, France. E-mail: vincent.rivasseau@gmail.com \\ 2 Centre de Physique Théorique, CNRS UMR 7644, Ecole Polytechnique, 91128 Palaiseau, France \\ 3 Institutul de Fizica si Inginerie Nucleara Horia Hulubei, P. O. Box MG-6, \\ 077125 Bucuresti-Magurele, Romania \\ 4 Max-Planck-Institut fur Mathematik, Vivatsgasse 7, 53111 Bonn, Germany
}

Received: 8 February 2008 / Accepted: 9 June 2008

Published online: 22 October 2008 - (C) Springer-Verlag 2008

\begin{abstract}
In this paper we propose a translation-invariant scalar model on the Moyal space. We prove that this model does not suffer from the UV/IR mixing and we establish its renormalizability to all orders in perturbation theory.
\end{abstract}

\section{Introduction and Motivation}

Space-time coordinates should no longer commute at the Planck scale where gravity should be quantized. This observation is a strong physical motivation for noncommutative geometry, a general mathematical framework developed by A. Connes and others [1]. Non-commutative field theory is the reformulation of ordinary quantum field theory on such a non-commutative background. It may represent a bridge between the current standard model of quantum fields on ordinary commutative $\mathbb{R}^{4}$ and a future formalism including quantum gravity which hopefully should be background independent.

Initially there was hope that non-commutative field theory would behave better in the ultraviolet regime [2]. Later motivation came from string theory, because field theory on simple non-commutative spaces (such as flat space with Moyal-Weyl product) appear as special effective regimes of the string $[3,4]$. Finally another very important motivation comes from the study of ordinary physics in strong external field (such as the quantum Hall effect) [5-7]. Such situations which have not been solved analytically with the ordinary commutative techniques may probably be studied more fruitfully with non-commutative techniques.

Renormalization is the soul of ordinary field theory and one would certainly want to extend it to the non-commutative setting. But the simplest non-commutative model, namely $\phi_{4}^{\star 4}$, whose action is given by (2.1) below, was found to be not renormalizable because of a surprising phenomenon called UV/IR mixing [8]. This mixing also occurs in non-commutative Yang-Mills theories. Roughly speaking the non-commutative theory still has infinitely many ultraviolet divergent graphs but fewer than the ordinary one. 
However some ultraviolet convergent two point graphs, such as the "non-planar tadpole" generate infrared divergences which are not of the renormalizable type ${ }^{1}$.

The first path out of this riddle came when H. Grosse and R. Wulkenhaar introduced a modified $\phi_{4}^{\star 4}$ model which is renormalizable $[10,11]$. They added to the usual propagator a marginal harmonic potential, which a posteriori appears required by Langmann-Szabo duality $\tilde{x}_{\mu}=2 \theta_{\mu \nu} x^{\nu} \leftrightarrow p_{\mu}[12]$.

The initial papers were improved and confirmed over the years through several independent methods $[13,14]$. The main property of the Grosse-Wulkenhaar model is that its $\beta$-function vanishes at all orders at the self-duality point $\Omega=1$ [15-17]. The exciting conclusion is that this model is asymptotically safe, hence free of any Landau ghost, and should be a fully consistent theory at the constructive level. This is because wave function renormalization exactly compensates the renormalization of the four-point function, so that the flow between the bare and the renormalized coupling is bounded.

Essentially most of the standard tools of field theory such as parametric $[18,19]$ and Mellin representations, [20] dimensional regularization and renormalization [21] and the Connes-Kreimer Hopf algebra formulation of renormalization [22] have now been generalized to renormalizable non-commutative quantum field theory. Other renormalizable models have been also developed such as the orientable Gross-Neveu model [23].

For a general recent review on non-commutative physics including these new developments on non-commutative field theory, see [24].

However there are two shortcomings of the Grosse-Wulkenhaar (GW) model. Firstly it breaks translation invariance so that its relevance to physics beyond the standard model would be indirect at best; one should either use more complicated "covariant" models with harmonic potentials which are invariant under "magnetic translations", such as the Langmann-Szabo-Zarembo model [25] or one should understand how many short distance localized GW models may glue into a translation-invariant effective model. Secondly it is not easy to generalize the GW method to gauge theories, which do present ultraviolet/infrared mixing. Trying to maintain both gauge invariance and LangmannSzabo duality one is lead to theories with non-trivial vacua [26-29], in which perturbation theory is difficult and renormalizability to all orders is therefore unclear up to now.

Motivated by these considerations we explore in this paper another solution to the ultraviolet infrared mixing for the $\phi_{4}^{\star 4}$ theory. It relies on the very natural idea to incorporate into the propagator the infrared mixing effects. This is possible because the sign of the mixing graphs is the right one. One can therefore modify the propagator to include from the start a $1 / p^{2}$ term besides the ordinary $p^{2}$ term, and to define new renormalization scales accordingly. Adding the interaction and expanding into the coupling constant we prove in this paper that the model modified in this way is indeed renormalizable at all orders of perturbation theory. This is because the former infrared effects now just generate a flow (in fact a finite flow) for the corresponding $1 / p^{2}$ term in the propagator. The "ordinary" $\phi_{4}^{\star 4}$ is formally recovered in the case where the bare coefficient of the $1 / p^{2}$ term is zero.

The advantages of this " $1 / p^{2}-\phi_{4}^{\star 4}$ " model are complementary to those of the GW model. The main advantage is that the model does not break translation invariance. The main inconvenience is that there is no analog of the Langmann-Szabo symmetry so that

\footnotetext{
1 This UV/IR mixing although quite generic may be avoided in some classes of "orientable models". Remark also that in Minkowski space if one maintains a rigorous notion of causality, there are strong indications that ultraviolet/infrared mixing does not occur [9]. However the Minkowski theory has complications of its own which make it harder to study.
} 
one should not expect this $\phi_{4}^{\star 4}$ model to make sense non-perturbatively. However the real interest of this work is perhaps to offer an alternative road to the solution of ultraviolet/infrared mixing in the case of gauge theories. It may lead to gauge and translation invariant models with trivial vacua. Remark that since ordinary non-Abelian gauge theories are asymptotically free, there is no real need for the non-commutative version to behave better than the commutative case. This removes some of the motivation to implement Langmann-Szabo duality in that case. Therefore we hope the model studied here may be a step towards a better global proposal for a non-commutative generalization of the standard model. This proposal may perhaps have to combine different solutions of the ultraviolet/infrared mixing in the Higgs and gauge sectors of the theory.

This model has already been used in [30]. Conjecturing that it would be renormalizable, the associated propagator (corresponding to Coulomb's law in a gauge theory) was proven to decay exponentially over a distance $\sqrt{\theta}$.

Let us finally comment on the physics of this model. The propagator we use deviates significantly from the usual commutative one in the infrared ${ }^{2}$. It is legitimate to ask whether commutative physics can be recovered as an effective limit of our model. If we couple the infrared and $\theta \rightarrow 0$ limits in a certain way, the singular part of the two point function becomes a mass counterterm. So letting the counterterms of the theory depend on $\theta$ we may reach a smooth commutative limit.

The paper is organized as follows. Section 2 recalls useful facts about Feynman graphs and defines our model. The main result of the paper, Theorem 2.1 below is stated at the end of that section. The proof is through the usual renormalization group multiscale analysis. The definition of the renormalization group slices and the power counting is given in Sect. 3 and the proof of the theorem is completed in Sect. 4 using the momentum representation. Finally some low order renormalized amplitudes for this theory are computed in Appendix A.

\section{Model and Main Result}

2.1. The "naive" $\phi^{\star 4}$ model. It is obtained by replacing the ordinary commutative action by the Moyal-Weyl $\star$-product

$$
S[\phi]=\int d^{4} x\left(\frac{1}{2} \partial_{\mu} \phi \star \partial^{\mu} \phi+\frac{1}{2} \mu^{2} \phi \star \phi+\frac{\lambda}{4 !} \phi \star \phi \star \phi \star \phi\right),
$$

with Euclidean metric. The commutator of two coordinates is

$$
\left[x^{\mu}, x^{\nu}\right]_{\star}=\imath \Theta^{\mu \nu},
$$

where

$$
\Theta=\left(\begin{array}{cccc}
0 & \theta & 0 & 0 \\
-\theta & 0 & 0 & 0 \\
0 & 0 & 0 & \theta \\
0 & 0 & -\theta & 0
\end{array}\right) .
$$

In momentum space the action (2.1) becomes

$$
S[\phi]=\int d^{4} p\left(\frac{1}{2} p_{\mu} \phi p^{\mu} \phi+\frac{1}{2} \mu^{2} \phi \phi+\frac{\lambda}{4 !} \phi \star \phi \star \phi \star \phi\right) .
$$

\footnotetext{
2 This is also the case of the Grosse-Wulkenhaar propagator.
} 
The propagator is the same as in the commutative case

$$
\frac{1}{p^{2}+\mu^{2}} \text {. }
$$

2.2. Feynman graphs: planarity and non-planarity, rosettes. In this subsection we give some useful conventions and definitions. Consider a $\phi^{\star 4}$ graph with $n$ vertices, $L$ internal lines and $F$ faces. One has

$$
2-2 g=n-L+F,
$$

where $g \in \mathbb{N}$ is the genus of the graph. If $g=0$ the graph is planar, if $g>0$ it is non-planar. Furthermore, we call a planar graph regular if it has a single face broken by external lines. We call $B$ the number of such faces broken by external lines.

The $\phi^{4}$ graphs also obey the relation

$$
L=\frac{1}{2}(4 n-N)
$$

where $N$ is the number of external legs of the graph.

In [31], T. Filk defined "contractions moves" on a Feynman graph. The first such move consists in reducing a tree line and gluing together the two vertices at its ends into a bigger one. Repeating this operation for the $n-1$ lines of a tree, one obtains a single final vertex with all the loop lines hooked to it - a rosette (see Fig. 1).

Note that the number of faces and the genus of the graph do not change under this operation. Furthermore, the external legs will break the same faces on the rosette. When one deals with a planar graph, there will be no crossing between the loop lines on the rosette. The example of Fig. 1 corresponds thus to a non-planar graph (one has crossings between e.g. the loop lines 3 and 5). Following [31] the rosette amplitude is

$$
\tilde{V} \text { (external momenta) } e^{\frac{l}{2} \sum_{i j} I_{i j} \Theta \mu \nu} k_{i}^{\mu} k_{j}^{\nu},
$$

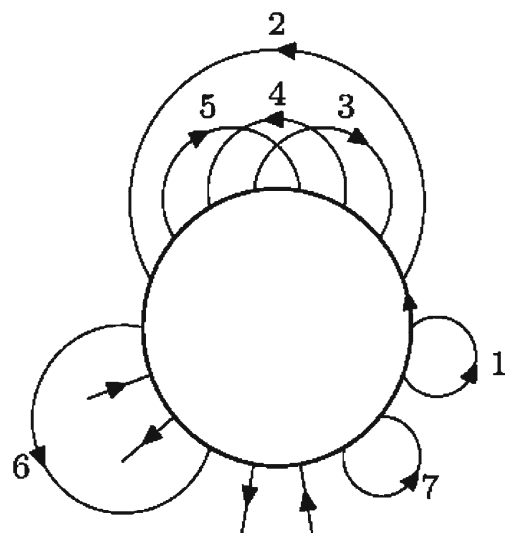

Fig. 1. An example of a rosette 


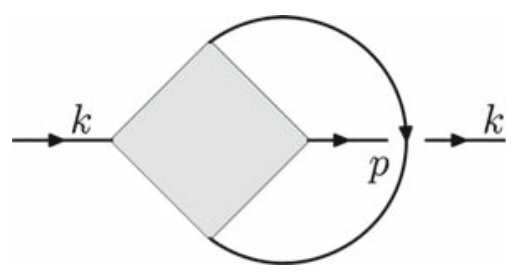

Fig. 2. The non-planar tadpole

where the intersection matrix $I_{i j}$ is given by

$$
I_{i j}=\left\{\begin{array}{l}
1, \text { if line } j \text { crosses line } i \text { from right, } \\
-1 \text { if line } j \text { crosses line } i \text { from left, } \\
0 \text { if lines } i \text { and } j \text { do not cross, }
\end{array}\right.
$$

where $i$ and $j$ correspond to an (arbitrary) numeration of the lines, independent of them being external or internal lines of the Feynman graph. An orientation is given by the sign convention chosen for the momenta in the conservation conditions. $\Theta$ is the non-commutativity matrix (see Eq. (2.3)). Furthermore the overall phase factor corresponding to the external momenta is

$$
\tilde{V}\left(k_{1}, \ldots, k_{N}\right)=\delta\left(k_{1}+\ldots+k_{N}\right) e^{\frac{l}{2} \sum_{i<j}^{N} k_{i}^{\mu} k_{j}^{\nu} \Theta_{\mu \nu}},
$$

which has exactly the form of a Moyal kernel.

2.3. UV/IR Mixing. The non-locality of the $\star$-product leads to a new type of divergence, the UV/IR mixing [8]. This can be seen already in the non-planar tadpole (see Fig. 2). Although this graph has zero genus, since it has two faces broken by external lines, it will lead to non-planarity when inserted into larger graphs.

The amplitude of this non-planar tadpole with internal momentum higher than the external momentum is up to a constant

$$
T=\int_{0}^{k^{-2}} d \alpha \int d^{4} p e^{\imath k \Theta p} e^{-\alpha\left(p^{2}+\mu^{2}\right)} .
$$

Integrating the Gaussian (and setting $\theta=1$ ) holds

$$
T=\int_{0}^{k^{-2}} \frac{d \alpha}{\alpha^{2}} e^{-\frac{k^{2}}{\alpha}} e^{-\alpha \mu^{2}}
$$

If $k>1$ then

$$
|T|<\int_{0}^{\infty} \frac{d \alpha}{\alpha^{2}} e^{-\frac{1}{\alpha}}=1 .
$$

Let $k<1$. We have

$$
T=\int_{0}^{k^{2}} \frac{d \alpha}{\alpha^{2}} e^{-\frac{k^{2}}{\alpha}} e^{-\alpha \mu^{2}}+\int_{k^{2}}^{k^{-2}} \frac{d \alpha}{\alpha^{2}} e^{-\frac{k^{2}}{\alpha}} e^{-\alpha \mu^{2}} .
$$


Rescaling $\alpha=k^{2} \beta$ we have for the first integral

$$
\frac{1}{k^{2}} \int_{0}^{1} \frac{d \beta}{\beta^{2}} e^{-\beta^{-1}} e^{-\beta k^{2} m^{2}}=\frac{F(k)}{k^{2}},
$$

with $F$ an analytic function of $k$.

We separate again the second integral as

$$
\int_{k^{2}}^{\mu^{-2}} \frac{d \alpha}{\alpha^{2}} e^{-\frac{k^{2}}{\alpha}} e^{-\alpha \mu^{2}}+\int_{\mu^{-2}}^{k^{-2}} \frac{d \alpha}{\alpha^{2}} e^{-\frac{k^{2}}{\alpha}} e^{-\alpha \mu^{2}} .
$$

The second integral is bounded by a constant uniformly in $k$. In the first integral we Taylor-expand $e^{-\alpha \mu^{2}}$ to get

$$
\int_{k^{2}}^{\mu^{-2}} \frac{d \alpha}{\alpha^{2}} e^{-\frac{k^{2}}{\alpha}}-\int_{k^{2}}^{\mu^{-2}} \frac{d \alpha}{\alpha^{2}} e^{-\frac{k^{2}}{\alpha}} \alpha \mu^{2}+\int_{k^{2}}^{\mu^{-2}} \frac{d \alpha}{\alpha^{2}} e^{-\frac{k^{2}}{\alpha}} O\left(\alpha^{2}\right) .
$$

The first term integrates to $k^{-2} F^{\prime}(k)$ with $F^{\prime}$ analytic, the second computes to $\mu^{2} \ln k^{2}+$ $F^{\prime \prime}(k)$ with $F^{\prime \prime}$ analytic and the third is uniformly bounded. Thus the tadpole is

$$
T=\frac{c}{k^{2}}+c^{\prime} \ln \left(k^{2}\right)+F(k)
$$

with $c$ and $c^{\prime}$ constants and $F$ an analytic function at $k=0$.

We note that for a non-massive model the second term vanishes. One can include the contribution of the non-planar tadpole in the complete two-point function to obtain a dressed propagator. This motivates a modification of the kinetic part of the action (2.4) which leads to

2.4. The $1 / p^{2} \phi_{4}^{\star 4}$ Model. This model is defined by the following action:

$$
S[\phi]=\int d^{4} p\left(\frac{1}{2} p_{\mu} \phi p^{\mu} \phi+\frac{1}{2} \mu^{2} \phi \phi+\frac{1}{2} a \frac{1}{\theta^{2} p^{2}} \phi \phi+\frac{\lambda}{4 !} \phi \star \phi \star \phi \star \phi\right),
$$

with $a$ some dimensionless parameter. The propagator is

$$
\frac{1}{p^{2}+\mu^{2}+\frac{a}{\theta^{2} p^{2}}},
$$

and we choose $a \geq 0$ so that this propagator is well-defined and positive.

Using (2.8) and (2.20) the amplitude of a $N$-point graph is written

$$
\begin{aligned}
\mathcal{A}(G)= & \delta\left(\sum_{i=1 \ldots N} k_{i}\right) e^{\frac{l}{2} \sum_{i<j}^{N} k_{i} \Theta k_{j}} \int \prod_{i=1}^{L} d^{4} p_{i} \frac{1}{p_{i}^{2}+\frac{a}{\theta^{2} p_{i}^{2}}+\mu^{2}} \\
& \times \prod_{v \neq \bar{v}} \delta\left(q_{1}^{v}+q_{2}^{v}+q_{3}^{v}+q_{3}^{v}\right) e^{\frac{l}{2} \sum_{i j} I_{i j} q_{i}^{v} \Theta q_{j}^{v}},
\end{aligned}
$$

with $k$ the external momenta, $p$ the internal momenta, $q^{v}$ a generic notation for internal and external momenta at vertex $v$, and $\bar{v}$ an external vertex of the graph chosen as root (to extract the global $\delta$ conservation on external momenta).

The main result of this paper is 
Theorem 2.1 (Main Result). The model defined by the action (2.19) is perturbatively renormalizable to all orders.

The proof is given in the next two sections. We proceed now to the usual RG analysis by defining slices and establishing power counting.

\section{Slices and Power counting}

In this section we establish the power counting of our model. For that purpose we shall use the very powerful tool of multiscale analysis. Power counting and the renormalization theory rely on some scale decomposition and the renormalization group is oriented: it integrates "fluctuating scales" (which we call here "high" scales) and computes an effective action for background scales (here called "low" scales). There are several technical different ways to define the RG scales, but in perturbation theory the best way is certainly to define the high scales as the locus where the denominator $D$ of the propagator is big and the low scales as the locus where it is small, cutting the slices into a geometric progression. This certainly works well for the very different RGs of ordinary statistical mechanics $\left(D=p^{2}\right)$, of condensed matter $\left(D=i p_{0}+(\boldsymbol{p})^{2}-1\right)$ and of the Grosse-Wulkenhaar model $\left(D=p^{2}+\Omega^{2} x^{2}\right)$. We use the same idea here again with $D=p^{2}+a / p^{2}$.

Power counting then evaluates contributions of connected subgraphs, also called "quasi local components" for which all internal scales are "higher" than all external scales in the sense above. We shall not rederive this basic principle here and shall use directly the particular version and notations of [32], in which these quasi-local components are labeled as $G_{r}^{j}$.

Before going into the detailed analysis of these contributions we first note a very important feature of our model: the term $a p^{-2}$ changes the UV and IR regions. For the rest of this paper we set $\theta=1$. We employ the Schwinger trick and write:

$$
\frac{1}{p^{2}+a p^{-2}+\mu^{2}}=\int_{0}^{\infty} e^{-\alpha\left(p^{2}+a p^{-2}+\mu^{2}\right)} d \alpha .
$$

Let $M>1$. Slice the propagators as

$$
\begin{aligned}
C(p) & =\sum_{i=0}^{\infty} C^{i}(p) \\
C^{i}(p) & =\int_{M^{-2 i}}^{M^{-2(i-1)}} d \alpha e^{-\alpha\left(p^{2}+a p^{-2}+\mu^{2}\right)} \leq K e^{-c M^{-2 i}\left(p^{2}+a p^{-2}+\mu^{2}\right)}, i \geq 1, \\
C^{0}(p) & =\int_{1}^{\infty} d \alpha e^{-\alpha\left(p^{2}+a p^{-2}+\mu^{2}\right)} \leq K e^{-c p^{2}}
\end{aligned}
$$

with $K$ and $c$ some constants which, for simplicity, will be omitted from now on. To the $i^{\text {th }}$ slice corresponds either a momentum $p \approx M^{i}$ or a momentum $p \approx M^{-i}$. Conversely, a momentum $k \approx M^{e}$ for $e \in \mathbb{Z}$ has a scale $\alpha=M^{-2|e|}$.

We have the following lemma: 
Lemma 3.1. The superficial degree of convergence $\omega(G)$ of a Feynman graph $G$ corresponding to the action (2.19) obeys

$$
\omega(G) \geq\left\{\begin{array}{ll}
N(G)-4, & \text { if } g(G)=0 \\
N(G)+4 & \text { if } g(G)>0
\end{array},\right.
$$

where $N(G)$ is the number of external legs of $G$, and $g(G)$ its genus.

Proof. The first line is easy. It is enough to take absolute values in (2.21), and apply the momentum routing. We briefly recall this procedure. We fix a scale attribution for all propagators. As the sum over the scales is easy to perform (along the same lines as in [32]) we concentrate on the problem of summing the internal momenta at fixed scale attribution $v$.

At any scale $i$ the graph $G^{i}$ made of lines with scales higher or equal to $i$ splits into $\rho$ connected components $G_{r}^{i}, r=1, \ldots, \rho$. We choose a spanning tree $T$ compatible with the scale attribution, that is each $T_{r}^{i}=T \cap G_{r}^{i}$ is a tree in the connected component $G_{r}^{i}$. We define the branch $b(l)$ associated to the tree line $l$ as the set of all vertices such that the unique path of lines connecting them to the root contains $l$. We can then solve the delta functions for the tree momenta as

$$
p_{l}=-\sum_{l^{\prime} \in b(l)} q_{l^{\prime}}
$$

where $l^{\prime} \in b(l)$ denotes all loops or external momenta touching a vertex in the branch $b(l)$. After integrating internal momenta we get the bound

$$
\mathcal{A}^{v} \leq \prod_{l} M^{-2 i_{l}} \prod_{l \in \mathcal{L}} M^{4 i_{l}}
$$

where $\mathcal{L}$ denotes the set of loop lines. The first factor comes from the prefactors of the propagators while the second comes from the integration of the loop momenta. We can reorganize the above product according to the scale attribution as

$$
\mathcal{A}^{\mathcal{v}} \leq \prod_{i, k} M^{-2 L\left(G_{r}^{i}\right)} M^{4\left[L\left(G_{r}^{i}\right)-n\left(G_{r}^{i}\right)+1\right]}=\prod_{i, k} M^{-\left[N\left(G_{r}^{i}\right)-4\right]},
$$

where we have used (2.7).

The second line of (3.3) is obtained using an argument similar to the one used in [14]. In fact if the graph is non-planar there will be two internal loop momenta $p$ and $q$ such that, after integrating all tree momenta with the delta functions, the amplitude contains a factor

$$
I=\int d^{4} p d^{4} q e^{-\alpha_{1} p^{2}-\alpha_{1} a p^{-2}-\alpha_{2} q^{2}-\alpha_{2} a q^{-2}+\imath p \wedge q} .
$$

A naive bound would be to bound the integral by $M^{4 i_{1}} M^{4 i_{2}}$. Instead we use

$$
\frac{1}{\left(1+M^{2 i_{1}} q^{2}\right)^{m}}\left(1+M^{2 i_{1}} \sum_{j} \frac{d^{2}}{d p_{j}^{2}}\right)^{m} e^{\imath p \wedge q}=e^{\imath p \wedge q} .
$$


Integrating by parts we get

$$
\begin{aligned}
|I| \leq & \int \frac{d^{4} p d^{4} q}{\left(1+M^{2 i_{1}} q^{2}\right)^{m}} e^{-M^{-2 i_{2}} q^{2}-M^{-2 i_{2}} q^{-2}} \\
& \times\left(1+M^{2 i_{1}} \sum_{j} \frac{d^{2}}{d p_{j}^{2}}\right)^{m} e^{-M^{-2 i_{1}} p^{2}-M^{-2 i_{1}} p^{-2}} .
\end{aligned}
$$

The derivative acting on the exponential gives factors of order at most $O(1)$. If we chose $m=3$ we have a bound

$$
I \leq K \int \frac{d^{4} p d^{4} q}{\left(1+M^{2 i_{1}} q^{2}\right)^{3}} e^{-M^{-2 i_{1}} p^{2}} \leq K^{\prime} .
$$

We have thus gained both factors $M^{4 i_{1}}$ and $M^{4 i_{2}}$ with respect to the naive bound.

\section{Renormalization}

We have established that all possible divergences come from planar 2 or 4 point graphs. Note that they may have more that one broken face ${ }^{3}$. We will prove that all divergences can be reabsorbed in a redefinition of the parameters in the action (2.19).

4.1. Two-point function. The single-broken-face 2-point graphs are ultraviolet divergent and as such give nontrivial mass and wave function renormalizations. By contrast the 2-point graphs with two broken faces are ultraviolet convergent. Nevertheless we will prove that they give a finite renormalization of the $1 / p^{2}$ term.

4.1.1. 2-point function with a single broken face. From the standard multiscale analysis we know that power counting has to be computed only for connected components of the $G_{r}^{j}$ type. Consider the case of such a planar, one particle irreducible, 2-point subgraph $S$ which is a component $G_{r}^{j}$ for $j$ for a certain range of slices $e<j \leq i$ between $e$, its highest external scale and $i$, its lowest internal scale (and a particular value of $r$ ),

$$
\begin{aligned}
\mathcal{A}\left(G_{r}^{j}\right)= & \delta\left(k_{1}+k_{2}\right) \int \prod_{l=1}^{L} d^{4} p_{l} \int_{M^{-2 i_{l}}}^{M^{-2\left(i_{l}-1\right)}} d \alpha_{l} e^{-\alpha_{l}\left[p_{l}^{2}+a p_{l}^{-2}+\mu^{2}\right]} \\
& \times \prod_{v \neq \bar{v}} \delta\left(q_{1}^{v}+q_{2}^{v}+q_{3}^{v}+q_{3}^{v}\right),
\end{aligned}
$$

where we consider that all eventual subrenormalizations have been performed. We perform the momentum routing for the subtree $T_{r}^{j}$. Let $k_{1}$ enter into the root vertex of $S$. We define

$$
T^{1}=\left\{l \in T \mid k_{2} \in b(l)\right\}, \quad T^{2}=T-T^{1}
$$

\footnotetext{
3 This stands in contrast with the Grosse-Wulkenhaar theory in which only graphs with a single broken face diverge.
} 
The amplitude is written, dropping the index on $k_{2}$ and forgetting the overall $\delta$ function,

$$
\begin{aligned}
\mathcal{A}(G)= & \int \prod_{l=1}^{|\mathcal{L}|} d^{4} p_{l} \prod_{l=1}^{L} \int_{M^{-2 i_{l}}}^{M^{-2\left(i_{l}-1\right)}} d \alpha_{l} \prod_{l \in \mathcal{L}} e^{-\alpha_{l}\left[p_{l}^{2}+a p_{l}^{-2}+\mu^{2}\right]} \\
& \times \prod_{l \in T^{2}} e^{-\alpha_{l}\left[\left(\sum_{l^{\prime} \in b(l)} p_{l^{\prime}}\right)^{2}+a\left(\sum_{l^{\prime} \in b(l)} p_{l^{\prime}}\right)^{-2}+\mu^{2}\right]} \\
& \times \prod_{l \in T^{1}} e^{-\alpha_{l}\left[\left(k+\sum_{l^{\prime} \in b(l)} p_{l^{\prime}}\right)^{2}+a\left(k+\sum_{l^{\prime} \in b(l)} p_{l^{\prime}}\right)^{-2}+\mu^{2}\right]} .
\end{aligned}
$$

We can now divide all integrals over $p_{l^{\prime}}$ 's in regions for each $l \in T_{1}$, according to whether $\left(\sum_{l^{\prime} \in b(l)} p_{l^{\prime}}\right)^{2}<a^{1 / 2}$ or $\left(\sum_{l^{\prime} \in b(l)} p_{l^{\prime}}\right)^{2} \geq a^{1 / 2}$. The regions with at least one condition $\left(\sum_{l^{\prime} \in b(l)} p_{l^{\prime}}\right)^{2}<a^{1 / 2}$ will count for $O(1)$ instead of $M^{4 i}$ and using directly the power counting argument we bound such a contribution to (4.3) by $M^{-2 i}$ per slice, for all $k$. In the following we will neglect all boundary terms on the sphere of radius $a^{1 / 2}$ as they are easy to bound uniformly in $k$. We conclude that only the case with all $\left(\sum_{l^{\prime} \in b(l)} p_{l^{\prime}}\right)^{2} \geq a^{1 / 2}$ have to be renormalized. In that case the factors $e^{-\alpha a\left(k+\sum_{l^{\prime} \in b(l)} p_{l^{\prime}}\right)^{-2}}$ give rise to an analytic behavior in $k$, and can be expanded around $k=0$.

We Taylor-expand the last line in (4.3) in that case. The odd terms in $p$ are zero after integration, as the branch momenta are linearly independent. For each term we have a development of the form

$$
e^{-\alpha_{l}\left[\left(\sum_{l^{\prime} \in b(l)} p_{l^{\prime}}\right)^{2}+a\left(\sum_{l^{\prime} \in b(l)} p_{l^{\prime}}\right)^{-2}+\mu^{2}\right]}\left(1-\alpha_{l} k^{2}+\alpha_{l}^{2} k^{4} \int_{0}^{1} d t(1-t) e^{-t \alpha_{l} k^{2}}\right) .
$$

Using the multiscale bound (3.2), we see that collecting the first terms we get a bound like (3.6), thus a quadratic mass divergence. If we have at least one factor in $\alpha_{l}$ we gain at least $M^{-2 i_{l}} \leq M^{-2 i}$ and we pay a factor $k^{2}$ which is of order $M^{2 e}$ because the external momenta is of scale $e$. Thus for all scales $j$ between $i$ and $e$ we have gained a factor $M^{-2}$ and the power counting factor associated to the corresponding connected component $G_{r}^{j}$, which was previously $M^{-\left(N\left(G_{r}^{j}\right)-4\right)}=M^{2}$, has become $M^{-\left(N\left(G_{r}^{j}\right)-2\right)}=1$. We get therefore a constant per slice as power counting for that connected component. As usual we recognize here the logarithmically divergent wave function renormalization associated to $S$. All other terms give convergent contributions, because a factor at least $M^{-4}$ per slice between $e$ and $i$ is gained.

4.1.2. 2-point function with two broken faces. The amplitude of a one-particle irreducible 2-point graph with two broken faces is

$$
\begin{aligned}
\mathcal{A}\left(G_{r}^{j}\right)= & \delta\left(k_{1}+k_{2}\right) \int \prod_{l=1}^{L} d^{4} p_{l} \int_{M^{-2 i_{l}}}^{M^{-2\left(i_{l}-1\right)}} d \alpha_{l} e^{-\alpha_{l}\left[p_{l}^{2}+a p_{l}^{-2}+\mu^{2}\right]} \\
& \times \prod_{v \neq \bar{v}} \delta\left(q_{1}^{v}+q_{2}^{v}+q_{3}^{v}+q_{3}^{v}\right) e^{l k_{2} \wedge\left(\sum_{l \in S} p_{l}\right)}
\end{aligned}
$$


with $S \in \mathcal{L}$ the set of loop lines crossed by the second external line. Performing again the momentum routing, dropping the index in $k_{2}$ and the global $\delta$ function yields

$$
\begin{aligned}
\mathcal{A}\left(G_{r}^{j}\right)= & \int \prod_{l=1}^{|\mathcal{L}|} d^{4} p_{l} \int_{M^{-2 i_{l}}}^{M^{-2\left(i_{l}-1\right)}} d \alpha_{l} \prod_{l \in \mathcal{L}} e^{-\alpha_{l}\left[p_{l}^{2}+a p_{l}^{-2}+\mu^{2}\right]} \\
& \times \prod_{l \in T^{2}} e^{-\alpha_{l}\left[\left(\sum_{l^{\prime} \in b(l)} p_{l^{\prime}}\right)^{2}+a\left(\sum_{l^{\prime} \in b(l)} p_{l^{\prime}}\right)^{-2}+\mu^{2}\right]} \\
& \times \prod_{l \in T^{1}} e^{-\alpha_{l}\left[\left(k+\sum_{l^{\prime} \in b(l)} p_{l^{\prime}}\right)^{2}+a\left(k+\sum_{l^{\prime} \in b(l)} p_{l^{\prime}}\right)^{-2}+\mu^{2}\right]} e^{l k \wedge\left(\sum_{l \in S} p_{l}\right)} .
\end{aligned}
$$

We use the same decomposition as before according to whether $\left(\sum_{l^{\prime} \in b(l)} p_{l^{\prime}}\right)^{2}<a^{1 / 2}$ or $\left(\sum_{l^{\prime} \in b(l)} p_{l^{\prime}}\right)^{2} \geq a^{1 / 2}$. Again only the case with all $\left(\sum_{l^{\prime} \in b(l)} p_{l^{\prime}}\right)^{2} \geq a^{1 / 2}$ is potentially divergent and could give rise to a non-analytic behavior in $k$.

We choose a line $l^{\prime} \in S$, use

$$
e^{l k \wedge\left(\sum_{l \in S} p_{l}\right)}=-\frac{1}{k^{2}} \Delta_{p_{l^{\prime}}} e^{\imath k \wedge\left(\sum_{l \in S} p_{l}\right)}
$$

and integrate by parts in (4.6) to get

$$
\begin{aligned}
\mathcal{A}\left(G_{r}^{j}\right)= & -\frac{1}{k^{2}} \int \prod_{l=1}^{|\mathcal{L}|} d^{4} p_{l} e^{l k \wedge\left(\sum_{l \in S} p_{l}\right)} \int_{M^{-2 i_{l}}}^{M^{-2\left(i_{l}-1\right)}} d \alpha_{l} \Delta_{p_{l^{\prime}}}\left(\prod_{l \in \mathcal{L}} e^{-\alpha_{l}\left[p_{l}^{2}+a p_{l}^{-2}+\mu^{2}\right]}\right. \\
& \times \prod_{l \in T^{2}} e^{-\alpha_{l}\left[\left(\sum_{l^{\prime} \in b(l)} p_{l^{\prime}}\right)^{2}+a\left(\sum_{l^{\prime} \in b(l)} p_{l^{\prime}}\right)^{-2}+\mu^{2}\right]} \\
& \left.\times \prod_{l \in T^{1}} e^{-\alpha_{l}\left[\left(k+\sum_{l^{\prime} \in b(l)} p_{l^{\prime}}\right)^{2}+a\left(k+\sum_{l^{\prime} \in b(l)} p_{l^{\prime}}\right)^{-2}+\mu^{2}\right]}\right)
\end{aligned}
$$

The derivatives acting on the Gaussian will give rise to insertions scaling like $\alpha, \alpha^{2} p^{2}$, $\alpha p^{-2}, \alpha p^{-4}, \alpha^{2} p^{-2}, \alpha^{2} p^{-4}$. The first two terms scale as $M^{-2 i}$ in a slice while the rest scale at least as $M^{-4 i}$. Using again the trick (4.7), and the power counting bound we get, when summing over all slices, a behavior like

$$
\mathcal{A}\left(G_{r}^{j}\right)=\frac{1}{k^{4}} \sum_{i=j}^{\infty} M^{-2 i}=\frac{1}{k^{2}} \frac{M^{-2 j}}{M^{2 e}} K,
$$

with $K$ some constant, if $k \approx M^{e}$ (and consequently of scale $|e|$ ). As the scale $j$ is ultraviolet with respect to $|e|$ we bound

$$
M^{-2 j-2 e} \leq M^{-2(|e|+e)} \leq 1 .
$$

We have thus proved that

$$
\mathcal{A}\left(G_{r}^{j}\right)=\frac{1}{k^{2}} F(k)
$$


with $F(k)$ a function uniformly bounded by a constant for all $k .^{4}$ We identify the terms $F(0)$ as a finite renormalization for the coefficient $a$ in the Lagrangian. Note that using this scale decomposition there are no logarithmic subleading divergences for this two point function with two broken faces.

4.2. Four-points function. The amplitude of a planar regular four-points graph is given by:

$$
\begin{aligned}
\mathcal{A}\left(G_{r}^{j}\right)= & \delta\left(k_{1}+k_{2}+k_{3}+k_{4}\right) e^{\frac{l}{2} \sum_{i<j} k_{i} \wedge k_{j}} \\
& \times \int \prod_{l=1}^{L} d^{4} p_{l} \int_{M^{-2 i_{l}}}^{M^{-2\left(i_{l}-1\right)}} d \alpha_{l} e^{-\alpha_{l}\left[p_{l}^{2}+a p_{l}^{-2}+\mu^{2}\right]} \prod_{v \neq \bar{v}} \delta\left(q_{1}^{v}+q_{2}^{v}+q_{3}^{v}+q_{3}^{v}\right) .
\end{aligned}
$$

The first line reproduces exactly the Moyal four-points kernel. Power counting leads to bound the second line by a constant per slice, thus it corresponds to a logarithmic divergence, which in turn generates a logarithmic coupling constant renormalization.

Some comments are in order for the planar four-points graphs with more than one broken face. Using (4.7) once, we get a bound like

$$
\mathcal{A}\left(G_{r}^{j}\right)=\frac{1}{k^{2}} \sum_{i=j}^{\infty} M^{-2 i}=\frac{M^{-2 j}}{M^{2 e}} K,
$$

and by (4.10) we see that the amplitude of such a graph is a function of external momenta uniformly bounded by some constant.

\section{Conclusions and Perspectives}

We have thus proved in this paper that the scalar model (2.19) is renormalizable at all orders in perturbation theory. The renormalization of the planar regular graphs goes along the same lines as the renormalization of the Euclidean $\phi^{4}$ on a 4-dimensional commutative space. The non-planar graphs remain convergent and the main difference concerns the planar irregular graphs. The comparison with the action (2.1) (which is non-renormalizable, with UV/IR mixing) and with the Grosse-Wulkenhaar model is summarized in the following table:

\begin{tabular}{|l|c|c|c|c|c|c|}
\hline & \multicolumn{3}{|c|}{ model $(2.1)$} & \multicolumn{2}{c|}{$G W$ model } & \multicolumn{2}{c|}{$1 / p^{2}-\phi_{4}^{\star 4}$ model } \\
& 2-points & 4-points & 2-points & 4-points & 2-points & 4-points \\
\hline planar regular & ren & ren & ren & ren & ren & ren \\
\hline planar irregular & UV/IR & log UV/IR & conv & conv & finite ren & conv \\
\hline non-planar & IR divergent & IR divergent & conv & conv & conv & conv \\
\hline
\end{tabular}

where ren means renormalizable.and conv means convergent.

Acknowledgement. We thank A. Abdesselam for indicating the proof of analyticity of $F$ in (4.11), and F. Vignes-Tourneret for useful comments. Furthermore, Adrian Tanasa gratefully acknowledges the European Science Foundation Research Networking Program "Quantum Geometry and Quantum Gravity" for the Short Visit Grants 2219 and 2232.

\footnotetext{
4 In fact $F(k)$ is analytic in $k$, as it is a sum of absolutely convergent integrals of analytic functions in $\alpha$ and $k$, in the region where all conditions $\left(\sum_{l^{\prime} \in b(l)} p_{l^{\prime}}\right)^{2} \geq a^{1 / 2}$ are fulfilled.
} 


\section{A. Examples of Graphs}

We illustrate the general results established in the previous section by some examples of two- and four-points graphs for which we analyze the Feynman amplitude.

A.1. A two-point graph example. Let us analyze the Feynman amplitude of the tadpole of Fig. 2. This graph has $g=0$ but $B=2$. Due to the new renormalization group slices the parameter $\alpha$ of an internal line obeys $\alpha<\min \left\{k^{2}, k^{-2}\right\}$. Therefore the amplitude of the non planar tadpole is (up to a constant)

$$
\int_{0}^{\min \left\{k^{2}, k^{-2}\right\}} d \alpha \int d^{4} p e^{i k \wedge p} e^{-\alpha\left(p^{2}+a p^{-2}+\mu^{2}\right)} .
$$

Applying (4.7) and integrating by parts holds:

$$
\begin{aligned}
& -\frac{1}{k^{2}} \int_{0}^{\min \left\{k^{2}, k^{-2}\right\}} d \alpha \int d^{4} p e^{i k \wedge p} \Delta_{p} e^{-\alpha\left(p^{2}+a p^{-2}+\mu^{2}\right)} \\
& =\frac{1}{k^{2}} \int_{0}^{\min \left\{k^{2}, k^{-2}\right\}} d \alpha \int d^{4} p e^{i k \wedge p} \\
& \quad \times\left(8 \alpha-\alpha^{2}\left(4 p^{2}-8 a p^{-2}+4 a^{2} p^{-6}\right)\right) e^{-\alpha\left(p^{2}+a p^{-2}+\mu^{2}\right)} .
\end{aligned}
$$

All but the first and second terms in (A.2) can be bounded by $k^{2}$ when taking absolute values such that the contribution to the amplitude of the tadpole is a constant. The coefficient of the $k^{-2}$ divergences is therefore

$$
c=\int_{0}^{\min \left\{k^{2}, k^{-2}\right\}} d \alpha \int d^{4} p e^{i k \wedge p}\left(8 \alpha+4 p^{2} \alpha^{2}\right) e^{-\alpha\left(p^{2}+a p^{-2}+\mu^{2}\right)} .
$$

Applying again 4.7 and integrating again by parts holds only terms like

$$
c_{n}=\frac{1}{k^{2}} \int_{0}^{\min \left\{k^{2}, k^{-2}\right\}} \alpha^{2} d \alpha \int d^{4} p e^{i k \wedge p}\left(\alpha p^{2}\right)^{n} e^{-\alpha\left(p^{2}+a p^{-2}+\mu^{2}\right)},
$$

with $n=0,1,2$. Taking absolute values, using $\left(\alpha p^{2}\right)^{n} e^{-\alpha p^{2}}<e^{-\frac{\alpha p^{2}}{2}}$ holds up to irrelevant constants,

$$
c_{n}<\frac{1}{k^{2}} \int_{0}^{\min \left\{k^{2}, k^{-2}\right\}} \alpha^{2} d \alpha \frac{1}{\alpha^{2}}=\frac{1}{k^{2}} \min \left\{k^{2}, k^{-2}\right\}<1 .
$$

We conclude that

$$
\frac{1}{k^{2}} F(k)+G(k)
$$

with $F$ and $G$ bounded and analytic at $k=0$. 


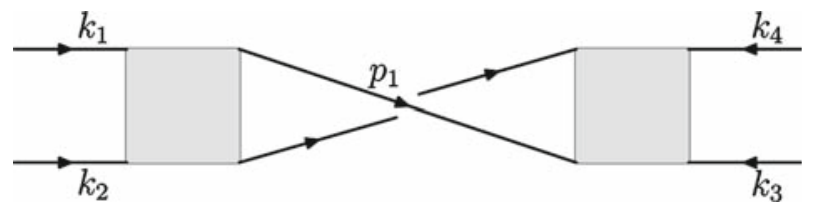

Fig. 3. An example of a four-point Feynman graph, planar but with 2 faces broken by external lines

A.2. Planar irregular four-points graphs. Take now the graph of Fig. 3. This graph has vanishing genus $(g=0)$ and two faces broken by external lines $(B=2)$.

The Feynman amplitude is written:

$$
\lambda^{2} \int d^{4} p_{1} e^{-2 i p_{1} \wedge\left(k_{1}+k_{2}\right)} \frac{1}{p_{1}^{2}+\mu^{2}+a \frac{1}{p_{1}^{2}}} \cdot \frac{1}{\left(p_{1}+k_{3}+k_{4}\right)^{2}+\mu^{2}+a \frac{1}{\left(p_{1}+k_{3}+k_{4}\right)^{2}}} .
$$

Let

$$
\begin{aligned}
& K=k_{1}+k_{2}=-\left(k_{3}+k_{4}\right), \\
& p_{2}=p_{1}+K .
\end{aligned}
$$

We now deal with the integral (2.7) as before, that is we use the Schwinger parametric representation and we express the oscillation factor using (4.7). Integrating by parts as above, one has

$$
-\frac{\lambda^{2}}{K^{2}} \int_{0}^{\min \left(K^{2}, K^{-2}\right)} d \alpha_{1} d \alpha_{2} \int d^{4} p_{1} e^{-2 i p_{1} \wedge K} \Delta_{p}\left[e^{-\alpha_{1}\left(p_{1}^{2}+a p_{1}^{-2}+\mu^{2}\right)} e^{-\alpha_{2}\left(p_{2}^{2}+a p_{2}^{-2}+\mu^{2}\right)}\right] .
$$

This further develops as:

$$
\begin{aligned}
& -\frac{\lambda^{2}}{K^{2}} \int_{0}^{\min \left(K^{2}, K^{-2}\right)} d \alpha_{1} d \alpha_{2} \int d^{4} p_{1} e^{-2 i p_{1} \wedge\left(k_{1}+k_{2}\right)} \\
& \quad\left[\left[-8 \alpha_{1}+\alpha_{1}^{2}\left(4 p_{1}^{2}+\frac{4 a^{2}}{p_{1}^{6}}-\frac{8 a}{p_{1}^{2}}\right)\right]+\left[-8 \alpha_{2}+\alpha_{2}^{2}\left(4 p_{2}^{2}+\frac{4 a^{2}}{p_{2}^{6}}-\frac{8 a}{p_{2}^{2}}\right)\right]\right. \\
& \left.+8 \alpha_{1} \alpha_{2}\left(p_{1 \mu}-\frac{a}{p_{1}^{4}} p_{1 \mu}\right)\left(p_{2}^{\mu}-\frac{a}{p_{2}^{4}} p_{2}^{\mu}\right)\right] e^{-\alpha_{1}\left(p_{1}^{2}+a p_{1}^{-2}+\mu^{2}\right)} e^{-\alpha_{2}\left(p_{2}^{2}+a p_{2}^{-2}+\mu^{2}\right)}
\end{aligned}
$$

Note that some of the terms above are of the same type as the ones appearing in (A.2) and can be bounded by $K^{2}$ when taking absolute values. Thus, their contribution to the amplitude is a constant. The rest of the terms of (A.10) can then be treated along the same lines as above. Take for example the second term of (A.10). This leads to an integral like

$$
\int_{0}^{\min \left(K^{2}, K^{-2}\right)} d \alpha_{1} d \alpha_{2} \alpha_{1} \frac{1}{\left(\alpha_{1}+\alpha_{2}\right)^{2}}
$$


One performs first the definite integral on $\alpha_{2}$. This leads to two terms which can be easily bounded by $K^{2}$. Finally, one concludes that the integral (A.10) leads to some constant result.

\section{References}

1. Connes, A.: Géometrie non commutative. Paris: InterEditions, 1990

2. Snyder, H.S.: Deformation quantization for actions of the affine group. Phys. Rev. 71, 38 (1947)

3. Connes, A., Douglas, M.R., Schwarz, A.: Noncommutative Geometry and Matrix Theory: Compactification on Tori. JHEP 9802, 3-43 (1998)

4. Seiberg, N., Witten, E.: String theory and noncommutative geometry. JHEP 9909, 32-131 (1999)

5. Susskind, L.: The Quantum Hall Fluid and Non-Commutative Chern Simons Theory. http://arxiv.org/list/ hep-th/0101029, 2001

6. Polychronakos, A.P.: Quantum Hall states on the cylinder as unitary matrix Chern-Simons theory. JHEP 06, 70-95 (2001)

7. Hellerman, S., Van Raamsdonk, M.: Quantum Hall physics equals noncommutative field theory. JHEP 10, 39-51 (2001)

8. Minwalla, S., Van Raamsdonk, M., Seiberg, N.: Noncommutative perturbative dynamics. JHEP 0002, 020 (2000)

9. Bahns, D.: The shuffle Hopf algebra and quasiplanar Wick products, conference proceedings. Non commutative Geometry and Physics, (Orsay, April 2007), http://arXiv.org/abs/0710.2787v1 [math.QA], 2007

10. Grosse, H., Wulkenhaar, R.: Power-counting theorem for non-local matrix models and renormalization. Commun. Math. Phys. 254, 91-127 (2005)

11. Grosse, H., Wulkenhaar, R.: Renormalization of $\phi^{4}$-theory on noncommutative $\mathbb{R}^{4}$ in the matrix base. Commun. Math. Phys. 256, 305-374 (2005)

12. Langmann, E., Szabo, R.J.: Duality in scalar field theory on noncommutative phase spaces. Phys. Lett. B 533, 168 (2002)

13. Rivasseau, V., Vignes-Tourneret, F., Wulkenhaar, R.: Renormalization of noncommutative $\phi_{4}^{\star 4}$-theory by multi-scale analysis. Commun. Math. Phys. 262, 565-594 (2006)

14. Gurau, R., Magnen, J., Rivasseau, V., Vignes-Tourneret, F.: Renormalization of non-commutative $\phi_{4}^{4}$ field theory in x space. Commun. Math. Phys. 267, 515 (2006)

15. Grosse, H., Wulkenhaar, R.: The beta-function in duality-covariant noncommutative $\phi^{4}$ theory. Eur. Phys. J. C 35, 277 (2004)

16. Disertori, M., Rivasseau, V.: Two and three loops beta function of non-commutative phi(4)**4 theory. Eur. Phys. J. C 50, 661 (2007)

17. Disertori, M., Gurau, R., Magnen, J., Rivasseau, V.: Vanishing of beta function of non-commutative $\phi_{4}^{4}$ theory to all orders. Phys. Lett. B 649, 95 (2007)

18. Gurău, R., Rivasseau, V.: Parametric representation of noncommutative field theory. Commun. Math. Phys. 272, 811 (2007)

19. Rivasseau, V., Tanasa, A.: Parametric representation of 'covariant' noncommutative QFT models. Commun. Math. Phys. 279(2), 355-379 (2008)

20. Gurau, R., Malbouisson, A.P.C., Rivasseau, V., Tanasa, A.: Non-Commutative Complete Mellin Representation for Feynman Amplitudes. http://arXiv.org/abs/0705.3437v1 [math-ph], 2007

21. Gurau, R., Tanasa, A.: Dimensional regularization and renormalization of non-commutative QFT. Submitted to Annales Henri Poincare, http://arXiv.org/abs/0706.1147v1 [math-ph], 2007

22. Tanasa, A., Vignes-Tourneret, F.: Hopf algebra of non-commutative field theory. Journ. Noncomm. Geom. 2, 125-139 (2008)

23. Vignes-Tourneret, F.: Renormalization of the orientable non-commutative Gross-Neveu model. Annales Henri Poincare 8, 427 (2007)

24. Duplantier, B., Rivasseau, V. eds.: Quantum Spaces, Progress in Mathematical Physics 53, Basel-Bosten: Birkhäuser, 2007

25. Langmann, E., Szabo, R.J., Zarembo, K.: Exact solution of quantum field theory on noncommutative phase spaces. JHEP 0401, 17-87 (2004)

26. de Goursac, A., Wallet, J.C., Wulkenhaar, R.: Noncommutative induced gauge theory. Eur. Phy. J. C 51, 977 (2007)

27. Grosse, H., Wohlgenannt, M.: Induced Gauge Theory on a Noncommutative Space. http://arXiv.org/list/ hep-th/0703169, 2007

28. Grosse, H., Wulkenhaar, R.: 8D-spectral triple on 4D-Moyal space and the vacuum of noncommutative gauge theory. http://arXiv.org/abs/0709.0095, V1 [hep-th], 2007 
29. de Goursac, A., Tanasa, A., Wallet, J.C.: Vacuum configurations for renormalizable non-commutative scalar models. Eur. Phys. J. C 53, 459 (2008)

30. Helling, R.C., You, J.: Macroscopic Screening of Coulomb Potentials From UV/IR-Mixing. http://arXiv. org/abs/0707.1885v.2 [hep-th], 2007

31. Filk, T.: Phys. Lett. B 376, 53 (1996)

32. Rivasseau, V.: From perturbative to Constructive Field Theory. Princeton, NJ: Princeton University Press, 1991

Communicated by M. Salmhofer 